\title{
Enhanced acousto-optic properties in layered media
}

\author{
M. J. A. Smith* and C. Martijn de Sterke \\ Centre for Ultrahigh bandwidth Devices for Optical Systems, Institute of Photonics and Optical Science, \\ School of Physics, The University of Sydney, NSW 2006, Australia \\ C. Wolff, M. Lapine, and C. G. Poulton \\ School of Mathematical and Physical Sciences, University of Technology Sydney, NSW 2007, Australia
}

(Received 6 February 2017; revised manuscript received 28 June 2017; published 23 August 2017)

\begin{abstract}
We present a rigorous procedure for evaluating the photoelastic coefficients of a layered medium in which the periodicity is smaller than the wavelengths of all optical and acoustic fields. Analytical expressions are given for the coefficients of a composite material comprising thin layers of optically isotropic materials. These photoelastic coefficients include artificial contributions that are unique to structured media and arise from the optical and mechanical contrast between the constituents. Using numerical examples, we demonstrate that the acousto-optic properties of layered structures can be enhanced beyond those of the constituent materials. Furthermore, we show that the acousto-optic response can be tuned as desired.
\end{abstract}

DOI: 10.1103/PhysRevB.96.064114

\section{INTRODUCTION}

Since the first phenomenological descriptions of the photoelastic effect by Pockels [1-3], acousto-optics has played a significant role in optics and materials science. Acousto-optic effects are critical for radio-frequency modulators [3-5], and the photoelastic effect is frequently used to determine stress distributions surrounding cracks and material defects [6]. More recently, acousto-optics has found applications in modern nanophotonics: photoelasticity is the fundamental effect that underpins cavity optomechanics $[7,8]$ and stimulated Brillouin scattering (SBS), which is critical for a diverse range of devices such as ultranarrow linewidth filters and high-resolution sensors $[5,9,10]$. These devices, however, rely on the existing, fixed, photoelastic response of the material platform, which in technologically important cases can be small $[11,12]$. At the same time, SBS is problematic for optical fiber systems [13], so there is considerable interest in both the suppression and the enhancement of photoelasticity, depending on the application.

It is well known that composite materials, such as layered media, can possess aggregate quantities that are markedly different from their constituents [14,15]. Recent work [16-18] has shown that this principle applies to the acousto-optic properties of composites. In contrast to the intricate and exotic designs seen in the optical metamaterials community, layered materials are among the simplest structures to fabricate, yet a complete picture of the acousto-optic properties of layered media has not yet been reported. To the best of our knowledge, the only other study concerning the photoelastic tensor of layered media is by Rouhani and Sapriel [19], where analytical expressions for an orthorhombic composite comprising orthorhombic layers were derived. However, nearly all of the expressions for the effective photoelastic coefficients are incomplete, as they do not include artificial photoelastic contributions (discussed below).

It has been widely accepted that acousto-optic interactions in uniform, nonpiezoelectric dielectric media are captured by

\footnotetext{
*michael.j.smith@sydney.edu.au
}

the photoelastic tensor $p_{i j k l}$ defined by

$$
\Delta\left(\varepsilon^{-1}\right)_{i j}=p_{i j k l} s_{k l},
$$

where $\Delta\left(\varepsilon^{-1}\right)_{i j}$ denotes a change in the inverse permittivity tensor and $s_{k l}$ is the linear strain tensor for small displacements from equilibrium. In this definition, the photoelastic tensor is treated as symmetric with respect to the first and second index pairs, i.e., $p_{i j k l}=p_{(i j)(k l)}$. However, the definition in (1) is sufficient to describe only the interaction between electromagnetic and acoustic waves in dielectrics possessing isotropic or cubic symmetry. This definition was sufficient in early research on light-sound interactions since the first solid materials examined either were of sufficiently high symmetry or possessed low optical anisotropy [5]. However, Nelson and Lax [20] established that this form of the photoelastic response omitted the contributions of local rotations that arise whenever acoustic shear waves propagate within the material; the effects of these local rotations on the permittivity tensor vanish for isotropic and cubic materials but are nonzero for media that possess lower levels of structural symmetry such as tetragonal lattices [21]. This roto-optic effect can be strong compared to the symmetric photoelastic effect and is directly related to the optical anisotropy of the material. The total photoelastic response of the material is given by [20]

$$
\begin{aligned}
\Delta\left(\varepsilon^{-1}\right)_{i j} & =P_{i j k l} \partial_{l} u_{k} \\
& =p_{i j k l} s_{k l}+r_{i j k l} r_{k l},
\end{aligned}
$$

where $P_{i j k l}$ is the full photoelastic tensor, $\partial_{l} u_{k}=s_{k l}+r_{k l}$ denotes the gradient of the displacement vector, $p_{(i j)(k l)}$ and $r_{(i j)[k l]}$ are the symmetric and antisymmetric components of $P_{i j k l}$, respectively, and $r_{k l}$ is the infinitesimal rotation tensor (where round- and square-bracket notations represent symmetric and antisymmetric index pairs, following Nelson and Lax [20], and we now omit bracket notation on index pairs for convenience). The definition (2) captures the potentially large influence that the antisymmetric component of the full photoelastic tensor (otherwise known as the roto-optic tensor) can have on the scattering of light by an acoustic shear wave [20]. 
The analytic form of the roto-optic tensor in uniform materials was given in $[3,20,22]$, where the tensor coefficients were found to be directly linked to the optical anisotropy of the medium (for materials that do not possess monoclinic or triclinic symmetry). Subsequently, it is important to consider the effects of both strains and rotations when studying acoustooptic interactions in optically anisotropic materials. Although a wide selection of natural uniform materials exhibits strong optical anisotropy (such as calcite [23]), it is also possible to achieve selective control over the optical birefringence of a medium by constructing composite materials [15].

In recent years, it has also been established that the photoelastic properties of structured materials exhibit a unique effect known as artificial photoelasticity. This effect was first recognized in composite materials comprising cubic arrays of spheres suspended in an otherwise uniform material by Smith et al. [16-18]. Artificial photoelasticity can be physically understood as follows: under an imposed strain, the different mechanical responses of the constituent materials alter the filling fraction and, in turn, contribute to changes in the permittivity of the composite. Such artificial contributions have been shown to play a significant role in the photoelastic properties of composites [17] and cannot be omitted, even for high-symmetry structured materials.

The two main contributions to an acousto-optic interaction are photoelasticity, describing changes in permittivity induced from bulk strains, and moving-boundary effects, describing permittivity changes due to boundary strains (e.g., the boundary between a waveguide or a cavity and the surrounding air) $[3,24]$. There is extensive literature examining interfacemotion (moving-boundary) contributions in acousto-optics for layered media [25-27], periodic structures [28,29], and general structures $[24,30]$, for example. However, the precise relationship between the moving-boundary effect and artificial photoelasticity is presently unclear. Both effects relate to interface motions, and both require a permittivity contrast in order to feature in an acousto-optic interaction. However, if the stiffness tensors of all layers are identical $C_{i j k l}=C_{i j k l}^{\prime}$, then artificial photoelasticity is zero, whereas moving-boundary contributions are not necessarily vanishing [24,25].

In place of photoelasticity and the moving-boundary effect, it is also possible to describe acousto-optic interactions in terms of electrostriction, which describes bulk stresses induced by an electromagnetic field, and radiation pressure, describing boundary stresses across dielectric interfaces [3]. Analytical expressions for the electrostrictive response of high symmetry structures (arrays of spheres), under the approximation that the shear contribution is negligible, were given in Smith et al. [16], and a rigorous numerical investigation followed soon after in Smith et al. [17,18]. In all instances, the electrostrictive properties of the composite were observed to be enhanced above and beyond the intrinsic electrostrictive properties of the constituents, indicating that strong effects may also be observed in structured materials with reduced symmetry, such as layered media.

In this paper, we derive the photoelastic coefficients of a layered medium, as shown in Fig. 1, giving the artificial contribution to the symmetric photoelastic tensor explicitly, in addition to an explicit representation for the roto-optic tensor. These expressions are obtained from the closed-

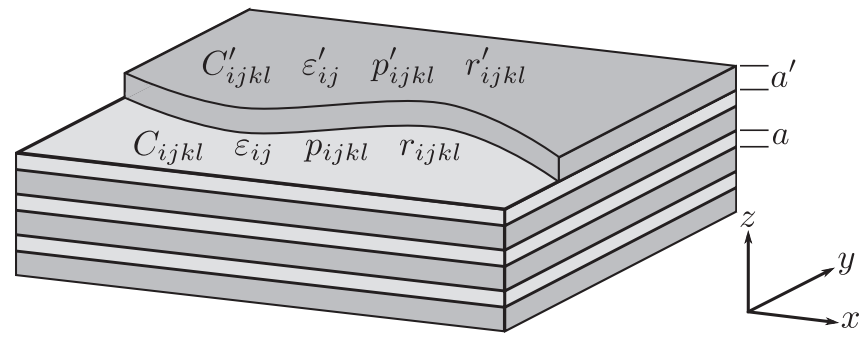

FIG. 1. Schematic of layered material investigated (infinitely extending in the $x-y$ plane) with periodicity along the $z$ axis and constituent parameters labeled.

form expressions for the effective permittivity and stiffness tensors, where we do not consider frequency dependence in the materials properties [31]. The procedure we outline for the effective permittivity tensor is a generalization of that presented in Bergman [32], which was extended to the effective stiffness tensor by Smith et al. [18], and is analogous to the approach by Grimsditch [33]. We demonstrate photoelastic coefficients with values above and beyond that of either constituent material, strong roto-optic coefficients, and non-negligible contributions from artificial photoelasticity for a silica-silicon and a silica-chalcogenide glass medium.

The outline of this paper is as follows. In Sec. II A we present the procedure for calculating the effective permittivity tensor $\varepsilon_{i j}^{\text {eff }}$. In Sec. II B we consider the analogous procedure for the effective stiffness tensor $C_{i j k l}^{\text {eff }}$. In Sec. II C we determine the symmetric photoelastic coefficients $p_{i j k l}^{\text {eff }}$, and in Sec. II D we give the antisymmetric photoelastic coefficients $r_{i j k l}^{\text {eff }}$. This section is followed by a numerical study of layered materials in Sec. III, before concluding remarks are given in Sec. IV.

\section{EFFECTIVE MATERIAL PARAMETERS}

In this section, we outline a compact procedure for calculating effective materials tensors, starting with the effective permittivity tensor $[18,32]$ and the effective stiffness tensor [18]. In this work, the layered medium is constructed as a one-dimensional stack of optically isotropic dielectric slabs, with periodicity in $z$, that forms a medium with tetragonal $(4 / \mathrm{mmm})$ symmetry [21], as shown in Fig. 1. Results for $p_{i j k l}$ are presented explicitly for this case, although the procedure is readily generalizable to consider layered materials made with optically anisotropic constituents.

The effective-medium procedure outlined here essentially replaces the layered material with a hypothetical effective material exhibiting the same boundary information on the edges of the unit cell and possessing the same energy as the layered material per unit cell. It is assumed that acousto-optic interactions are the only nonlinear effect that the effective medium exhibits. In the derivation that follows, we use the convention of unprimed notation for the first layer in the unit cell, primed notation for the second layer, and "eff" for the effective medium. It is assumed that the thicknesses of the two layers, $a$ and $a^{\prime}$, are small relative to the wavelength of all electromagnetic and acoustic fields (see Fig. 1). In other words, we examine the intrinsic bulk properties of the material in both the optical and acoustic long-wavelength regimes. 
It is also assumed that the optical and acoustic contrast between layers does not induce a perturbation to the magnetic field, i.e., $\mu_{i j}=\mu_{i j}^{\prime}=\mu_{i j}^{\text {eff }}=\delta_{i j}$, where $\delta_{i j}$ denotes the Kronecker delta and $\mu_{i j}$ is the relative permeability.

\section{A. Effective permittivity tensor}

We begin by computing the effective permittivity tensor for a layered medium and impose conventional electromagnetic boundary conditions across the layers; continuity of the tangential $\mathbf{E}$ field and normal $\mathbf{D}$ field for our layered medium requires that

$$
E_{\mathrm{x}}=E_{\mathrm{x}}^{\prime}, \quad E_{\mathrm{y}}=E_{\mathrm{y}}^{\prime}, \quad D_{\mathrm{z}}=D_{\mathrm{z}}^{\prime},
$$

where we further impose that the effective medium must take the same static field values at all boundaries,

$$
E_{\mathrm{x}}^{\mathrm{eff}}=E_{\mathrm{x}}=E_{\mathrm{x}}^{\prime}, \quad E_{\mathrm{y}}^{\mathrm{eff}}=E_{\mathrm{y}}=E_{\mathrm{y}}^{\prime}, \quad D_{\mathrm{z}}^{\mathrm{eff}}=D_{\mathrm{z}}=D_{\mathrm{z}}^{\prime} .
$$

We then require that the effective energy density [34]

$$
\mathcal{U}^{\text {eff }}=\frac{1}{2} E_{i}^{\mathrm{eff}} D_{i}^{\mathrm{eff}}
$$

is equivalent to the total energy density over the unit cell

$$
\mathcal{U}=\frac{1}{2}\left[f E_{i} D_{i}+(1-f) D_{i}^{\prime} E_{i}^{\prime}\right],
$$

where $f=a /\left(a+a^{\prime}\right)$ is the volume filling fraction, which gives rise to

$$
\begin{aligned}
& D_{\mathrm{x}}^{\mathrm{eff}}=f D_{\mathrm{x}}+(1-f) D_{\mathrm{x}}^{\prime}, \\
& D_{\mathrm{y}}^{\mathrm{eff}}=f D_{\mathrm{y}}+(1-f) D_{\mathrm{y}}^{\prime}, \\
& E_{\mathrm{z}}^{\mathrm{eff}}=f E_{\mathrm{z}}+(1-f) E_{\mathrm{z}}^{\prime} .
\end{aligned}
$$

Using (4) and (7) with the constitutive relations

$$
D_{i}^{\mathrm{eff}}=\varepsilon_{0} \varepsilon_{i j}^{\mathrm{eff}} E_{j}^{\mathrm{eff}}, \quad D_{i}=\varepsilon_{0} \varepsilon_{i j} E_{j}, \quad D_{i}^{\prime}=\varepsilon_{0} \varepsilon_{i j}^{\prime} E_{j}^{\prime},
$$

where $\varepsilon_{i j}$ denotes the permittivity tensor and $\varepsilon_{0}$ is the vacuum permittivity, it follows almost immediately that

$$
\begin{aligned}
\varepsilon_{\mathrm{xx}}^{\mathrm{eff}}= & f \varepsilon_{\mathrm{xx}}+(1-f) \varepsilon_{\mathrm{xx}}^{\prime}-\frac{f(1-f)\left(\varepsilon_{\mathrm{xz}}-\varepsilon_{\mathrm{xz}}^{\prime}\right)^{2}}{f \varepsilon_{\mathrm{zz}}^{\prime}+(1-f) \varepsilon_{\mathrm{zz}}}, \\
\varepsilon_{\mathrm{yy}}^{\mathrm{eff}}= & f \varepsilon_{\mathrm{yy}}+(1-f) \varepsilon_{\mathrm{yy}}^{\prime}-\frac{f(1-f)\left(\varepsilon_{\mathrm{yz}}-\varepsilon_{\mathrm{yz}}^{\prime}\right)^{2}}{f \varepsilon_{\mathrm{zz}}^{\prime}+(1-f) \varepsilon_{\mathrm{zz}}}, \\
\frac{1}{\varepsilon_{\mathrm{zz}}^{\mathrm{eff}}}= & \frac{f}{\varepsilon_{\mathrm{zz}}}+\frac{(1-f)}{\varepsilon_{\mathrm{zz}}^{\prime}} \\
\varepsilon_{\mathrm{yz}}^{\mathrm{eff}}= & \frac{f \varepsilon_{\mathrm{yz}} \varepsilon_{\mathrm{zz}}^{\prime}+(1-f) \varepsilon_{\mathrm{yz}}^{\prime} \varepsilon_{\mathrm{zz}}}{f \varepsilon_{\mathrm{zz}}^{\prime}+(1-f) \varepsilon_{\mathrm{zz}}}, \\
\varepsilon_{\mathrm{xz}}^{\mathrm{eff}}= & \frac{f \varepsilon_{\mathrm{xz}} \varepsilon_{\mathrm{zz}}^{\prime}+(1-f) \varepsilon_{\mathrm{xz}}^{\prime} \varepsilon_{\mathrm{zz}}}{f \varepsilon_{\mathrm{zz}}^{\prime}+(1-f) \varepsilon_{\mathrm{zz}}}, \\
\varepsilon_{\mathrm{xy}}^{\mathrm{eff}}= & f \varepsilon_{\mathrm{xy}}+(1-f) \varepsilon_{\mathrm{xy}}^{\prime} \\
& -\frac{f(1-f)\left(\varepsilon_{\mathrm{xz}}-\varepsilon_{\mathrm{xz}}^{\prime}\right)\left(\varepsilon_{\mathrm{yz}}-\varepsilon_{\mathrm{yz}}^{\prime}\right)}{f \varepsilon_{\mathrm{zz}}^{\prime}+(1-f) \varepsilon_{\mathrm{zz}}} .
\end{aligned}
$$

The expressions for $\varepsilon_{i j}^{\text {eff }}$ above are equivalent to those presented in Rouhani and Sapriel [19] and are valid for layered materials comprising fully anisotropic layers. Despite the equivalence of certain $\varepsilon_{i j}^{\text {eff }}$ coefficients in a tetragonal material (i.e., $\varepsilon_{\mathrm{xx}}^{\mathrm{eff}}=\varepsilon_{\mathrm{yy}}^{\mathrm{eff}}$ and $\varepsilon_{\mathrm{yz}}^{\mathrm{eff}}=\varepsilon_{\mathrm{xz}}^{\mathrm{eff}}=\varepsilon_{\mathrm{xy}}^{\mathrm{eff}}=0$ ), all permittivity coefficients are required to determine the photoelastic coefficients for the composite in Sec. II C. For reference, we represent elements of an inverse tensor by $\left(\varepsilon^{-1}\right)_{i j}$ and reciprocal values by $1 / \varepsilon_{i j}$.

\section{B. Effective stiffness tensor}

We now obtain closed-form expressions for the stiffness tensor of a layered material and begin by imposing conventional acoustic boundary conditions [35]: continuity of transverse velocity (or transverse displacement for timeharmonic fields in the long-wavelength limit) and continuity of the normal component of the stress field, which requires that

$$
u_{\mathrm{x}}=u_{\mathrm{x}}^{\prime}, \quad u_{\mathrm{y}}=u_{\mathrm{y}}^{\prime},
$$

in addition to

$$
\sigma_{\mathrm{xz}}=\sigma_{\mathrm{xz}}^{\prime}, \quad \sigma_{\mathrm{yz}}=\sigma_{\mathrm{yz}}^{\prime}, \quad \sigma_{\mathrm{zz}}=\sigma_{\mathrm{zz}}^{\prime},
$$

respectively. We then impose that the effective displacement and effective stress fields possess the same static values at the boundary as per the conditions above, for example, $u_{\mathrm{x}}^{\text {eff }}=$ $u_{\mathrm{x}}=u_{\mathrm{x}}^{\prime}$ and $\sigma_{\mathrm{xz}}^{\mathrm{eff}}=\sigma_{\mathrm{xz}}=\sigma_{\mathrm{xz}}^{\prime}$. In analogy with Sec. II A, we require that the strain energy density for the effective medium

$$
\mathcal{U}_{\mathrm{s}}^{\text {eff }}=\frac{1}{2} \sigma_{i j}^{\text {eff }} s_{i j}^{\text {eff }}
$$

is equivalent to the total strain energy density

$$
\mathcal{U}_{\mathrm{s}}=\frac{1}{2}\left[f \sigma_{i j} s_{i j}+(1-f) \sigma_{i j}^{\prime} s_{i j}^{\prime}\right],
$$

where $s_{i j}=\frac{1}{2}\left(\partial_{i} u_{j}+\partial_{j} u_{i}\right)$. This is satisfied provided

$$
\begin{aligned}
\partial_{\mathrm{x}} u_{\mathrm{z}}^{\mathrm{eff}} & =f \partial_{\mathrm{x}} u_{\mathrm{z}}+(1-f) \partial_{\mathrm{x}} u_{\mathrm{z}}^{\prime}, \\
\sigma_{\mathrm{xx}}^{\mathrm{eff}} & =f \sigma_{\mathrm{xx}}+(1-f) \sigma_{\mathrm{xx}}^{\prime}, \\
\partial_{\mathrm{y}} u_{\mathrm{z}}^{\mathrm{eff}} & =f \partial_{\mathrm{y}} u_{\mathrm{z}}+(1-f) \partial_{\mathrm{y}} u_{\mathrm{z}}^{\prime}, \\
\sigma_{\mathrm{yy}}^{\mathrm{eff}} & =f \sigma_{\mathrm{yy}}+(1-f) \sigma_{\mathrm{yy}}^{\prime}, \\
\partial_{\mathrm{z}} u_{\mathrm{z}}^{\mathrm{eff}} & =f \partial_{\mathrm{z}} u_{\mathrm{z}}+(1-f) \partial_{\mathrm{z}} u_{\mathrm{z}}^{\prime}, \\
\sigma_{\mathrm{xy}}^{\mathrm{eff}} & =f \sigma_{\mathrm{xy}}+(1-f) \sigma_{\mathrm{xy}}^{\prime},
\end{aligned}
$$

where, for convenience, we now differentiate (10) and compile these with the derivatives of the displacement fields in (14) along with the stress fields to obtain

$$
\begin{array}{ll}
\sigma_{\mathrm{xx}}^{\mathrm{eff}}=f \sigma_{\mathrm{xx}}+(1-f) \sigma_{\mathrm{xx}}^{\prime}, & s_{\mathrm{xx}}^{\mathrm{eff}}=s_{\mathrm{xx}}=s_{\mathrm{xx}}^{\prime}, \\
\sigma_{\mathrm{yy}}^{\mathrm{eff}}=f \sigma_{\mathrm{yy}}+(1-f) \sigma_{\mathrm{yy}}^{\prime}, & s_{\mathrm{yy}}^{\mathrm{eff}}=s_{\mathrm{yy}}=s_{\mathrm{yy}}^{\prime}, \\
\sigma_{\mathrm{zz}}^{\mathrm{eff}}=\sigma_{\mathrm{zz}}=\sigma_{\mathrm{zz}}^{\prime}, & s_{\mathrm{zz}}^{\mathrm{eff}}=f s_{\mathrm{zz}}+(1-f) s_{\mathrm{zz}}^{\prime}, \\
\sigma_{\mathrm{yz}}^{\mathrm{eff}}=\sigma_{\mathrm{yz}}=\sigma_{\mathrm{yz}}^{\prime}, & s_{\mathrm{yz}}^{\mathrm{eff}}=f s_{\mathrm{yz}}+(1-f) s_{\mathrm{yz}}^{\prime}, \\
\sigma_{\mathrm{xz}}^{\mathrm{eff}}=\sigma_{\mathrm{xz}}=\sigma_{\mathrm{xz}}^{\prime}, & s_{\mathrm{xz}}^{\mathrm{eff}}=f s_{\mathrm{xz}}+(1-f) s_{\mathrm{xz}}^{\prime}, \\
\sigma_{\mathrm{xy}}^{\mathrm{eff}}=f \sigma_{\mathrm{xy}}+(1-f) \sigma_{\mathrm{xy}}^{\prime}, & s_{\mathrm{xy}}^{\mathrm{eff}}=s_{\mathrm{xy}}=s_{\mathrm{xy}}^{\prime} .
\end{array}
$$

Using (15) along with the constitutive relations

$$
\sigma_{i j}^{\mathrm{eff}}=C_{i j k l}^{\mathrm{eff}} s_{k l}^{\mathrm{eff}}, \quad \sigma_{i j}=C_{i j k l} s_{k l}, \quad \sigma_{i j}^{\prime}=C_{i j k l}^{\prime} s_{k l}^{\prime},
$$

where $C_{i j k l}$ denotes the linear stiffness tensor, we recover the effective stiffness coefficients. Here the layers comprise 
optically isotropic media, from which we obtain all six unique nonvanishing parameters of the mechanical stiffness tensor $C_{i j k l}^{\text {eff }}$ for an effective tetragonal $(4 / \mathrm{mmm})$ material [21] as

$$
\begin{aligned}
C_{\mathrm{xxxx}}^{\mathrm{eff}}= & f C_{\mathrm{xxxx}}+(1-f) C_{\mathrm{xxxx}}^{\prime} \\
& -\frac{f(1-f)\left(C_{\mathrm{xxyy}}-C_{\mathrm{xxyy}}^{\prime}\right)^{2}}{f C_{\mathrm{xxxx}}^{\prime}+(1-f) C_{\mathrm{xxxx}}}, \\
C_{\mathrm{xxyy}}^{\mathrm{eff}}= & f C_{\mathrm{xxyy}}+(1-f) C_{\mathrm{xxyy}}^{\prime} \\
& -\frac{f(1-f)\left(C_{\mathrm{xxyy}}-C_{\mathrm{xxyy}}^{\prime}\right)^{2}}{f C_{\mathrm{xxxx}}^{\prime}+(1-f) C_{\mathrm{xxxx}}}, \\
C_{\mathrm{xxzz}}^{\mathrm{eff}}= & \frac{f C_{\mathrm{xxyy}} C_{\mathrm{xxxx}}^{\prime}+(1-f) C_{\mathrm{xxxx}} C_{\mathrm{xxyy}}^{\prime}}{f C_{\mathrm{xxxx}}^{\prime}+(1-f) C_{\mathrm{xxxx}}}, \\
\frac{1}{C_{\mathrm{zzzz}}^{\mathrm{eff}}=} & \frac{f}{C_{\mathrm{xxxx}}}+\frac{(1-f)}{C_{\mathrm{xxxx}}^{\prime}}, \\
\frac{1}{C_{\mathrm{yzyz}}^{\mathrm{eff}}}= & \frac{f}{C_{\mathrm{yzyz}}}+\frac{(1-f)}{C_{\mathrm{yzyz}}^{\prime}}, \\
C_{\mathrm{xyxy}}^{\mathrm{eff}}= & f C_{\mathrm{yzyz}}+(1-f) C_{\mathrm{yzyz}}^{\prime} .
\end{aligned}
$$

The expressions in (17) are equivalent to those presented in Rouhani and Sapriel [19] and Grimsditch [33], after considering the symmetry properties of the constituent layers.

\section{Effective symmetric photoelastic tensor}

In this section we evaluate the symmetric photoelastic tensor $p_{i j k l}^{\text {eff }}$ defined by

$$
\Delta\left(\varepsilon_{\text {eff }}^{-1}\right)_{i j}=p_{i j k l}^{\text {eff }} s_{k l}^{\text {eff }},
$$

or, equivalently,

$$
\Delta\left(\varepsilon_{\mathrm{eff}}\right)_{i j}=-\varepsilon_{i i}^{\mathrm{eff}} \varepsilon_{j j}^{\mathrm{eff}} p_{i j k l}^{\mathrm{eff}} s_{k l}^{\mathrm{eff}},
$$

provided the medium does not possess triclinic or monoclinic symmetry [22]. Expressions for the effective photoelastic tensor elements $p_{i j k l}^{\text {eff }}$ are obtained by differentiating the effective permittivity expressions $\varepsilon_{i j}^{\text {eff }}$ in (9) with respect to individual strain fields $s_{i j}^{\text {eff }}$ while holding other effective strain fields constant. For example, (18a) for an effective tetragonal $(4 / \mathrm{mmm})$ material is given by

$$
\Delta \varepsilon_{\mathrm{zz}}^{\mathrm{eff}}=-\left(\varepsilon_{\mathrm{zz}}^{\mathrm{eff}}\right)^{2}\left[p_{\mathrm{zzxx}}^{\mathrm{eff}} s_{\mathrm{xx}}^{\mathrm{eff}}+p_{\mathrm{zzxx}}^{\mathrm{eff}} s_{\mathrm{yy}}^{\mathrm{eff}}+p_{\mathrm{zzzz}}^{\mathrm{eff}} s_{\mathrm{zz}}^{\mathrm{eff}}\right],
$$

and subsequently,

$$
\begin{aligned}
& \left.\frac{\partial \varepsilon_{\mathrm{zz}}^{\mathrm{eff}}}{\partial s_{\mathrm{xx}}^{\mathrm{eff}}}\right|_{s_{\mathrm{yy}}^{\text {eff }}, s_{\mathrm{zz}}^{\text {eff }}}=-\left(\varepsilon_{\mathrm{zz}}^{\text {eff }}\right)^{2} p_{\mathrm{zzxx}}^{\text {eff }}, \\
& \left.\frac{\partial \varepsilon_{\mathrm{zz}}^{\mathrm{eff}}}{\partial s_{\mathrm{yy}}^{\mathrm{eff}}}\right|_{s_{\mathrm{xx}}^{\text {eff }}, s_{\mathrm{zz}}^{\text {eff }}}=-\left(\varepsilon_{\mathrm{zz}}^{\mathrm{eff}}\right)^{2} p_{\mathrm{zzxx}}^{\mathrm{eff}}, \\
& \left.\frac{\partial \varepsilon_{\mathrm{zz}}^{\mathrm{eff}}}{\partial s_{\mathrm{zz}}^{\text {eff }}}\right|_{s_{\mathrm{xx}}^{\text {eff }}, s_{\mathrm{yy}}^{\text {eff }}}=-\left(\varepsilon_{\mathrm{zz}}^{\text {eff }}\right)^{2} p_{\mathrm{zzzz}}^{\text {eff }} .
\end{aligned}
$$

Therefore, an analytical expression for $p_{\mathrm{zzzz}}^{\text {eff }}$ is recovered by differentiating $\varepsilon_{\mathrm{zz}}^{\text {eff }}(9 \mathrm{c})$ with respect to $s_{\mathrm{zz}}^{\text {eff }}$, with both $s_{\mathrm{xx}}^{\text {eff }}$ and $s_{\text {yy }}^{\text {eff }}$ held constant. The resulting expressions are then reduced using the tensor definitions for the constituent materials

$$
\begin{aligned}
& \Delta \varepsilon_{i j}=-\varepsilon_{i i} \varepsilon_{j j} p_{i j k l} s_{k l}, \\
& \Delta \varepsilon_{i j}^{\prime}=-\varepsilon_{i i}^{\prime} \varepsilon_{j j}^{\prime} p_{i j k l}^{\prime} s_{k l}^{\prime},
\end{aligned}
$$

the mechanical constitutive relations (16), and the mechanical boundary conditions in (15). We remark that the permittivities $\varepsilon_{i j}$ and $\varepsilon_{i j}^{\prime}$ are functions of their constituent strain fields alone [see Eq. (21)]. The derivation for all seven unique nonvanishing photoelastic constants necessary to describe a layered structure is extensive, and we feel that there is little merit in providing a complete outline for all terms. Accordingly, we consider the derivations for $p_{\mathrm{zzxx}}^{\text {eff }}$ and $p_{\mathrm{zzzz}}^{\text {eff }}$ alone and present the final expressions for all remaining coefficients.

As identified in (20a) above, we now implicitly differentiate the effective permittivity expression (9c) with respect to $s_{\mathrm{xx}}^{\text {eff }}$, holding the strain fields $s_{\mathrm{yy}}^{\text {eff }}$ and $s_{\mathrm{zz}}^{\text {eff }}$ constant, which admits

$$
\begin{aligned}
\left.\frac{1}{\left(\varepsilon_{\mathrm{zz}}^{\mathrm{eff}}\right)^{2}} \frac{\partial \varepsilon_{\mathrm{zz}}^{\mathrm{eff}}}{\partial s_{\mathrm{xx}}^{\mathrm{eff}}}\right|_{s_{\mathrm{yy}}^{\mathrm{eff}}, s_{\mathrm{zz}}^{\text {eff }}}= & \left.\frac{f}{\left(\varepsilon_{\mathrm{zz}}\right)^{2}} \frac{\partial \varepsilon_{\mathrm{zz}}}{\partial s_{\mathrm{xx}}^{\mathrm{eff}}}\right|_{s_{\mathrm{yy}}^{\mathrm{eff}}, s_{\mathrm{zz}}^{\text {eff }}}+\left.\frac{(1-f)}{\left(\varepsilon_{\mathrm{zz}}^{\prime}\right)^{2}} \frac{\partial \varepsilon_{\mathrm{zz}}^{\prime}}{\partial s_{\mathrm{xx}}^{\mathrm{eff}}}\right|_{s_{\mathrm{yy}}^{\text {eff }}, s_{\mathrm{zz}}^{\text {eff }}} \\
& -\left.\left(\frac{1}{\varepsilon_{\mathrm{zz}}}-\frac{1}{\varepsilon_{\mathrm{zz}}^{\prime}}\right) \frac{\partial f}{\partial s_{\mathrm{xx}}^{\mathrm{eff}}}\right|_{s_{\mathrm{yy}}^{\mathrm{eff}}, s_{\mathrm{zz}}^{\mathrm{eff}}} \cdot
\end{aligned}
$$

The first derivative in (22) follows immediately from the definition in (20a). The next derivative is evaluated using the definition of the photoelastic tensor (21a) in the first optically isotropic layer. An application of chain rule then admits

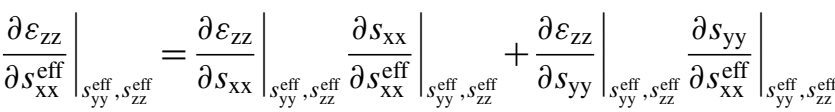

$$
\begin{aligned}
& +\left.\left.\frac{\partial \varepsilon_{\mathrm{zz}}}{\partial s_{\mathrm{zZ}}}\right|_{s_{\mathrm{yy}}^{\mathrm{eff}}, s_{\mathrm{zz}}^{\mathrm{eff}}} \frac{\partial s_{\mathrm{zz}}}{\partial s_{\mathrm{xx}}^{\mathrm{eff}}}\right|_{s_{\mathrm{yy}}^{\mathrm{eff}}, s_{\mathrm{zz}}^{\mathrm{eff}}} .
\end{aligned}
$$

Using the acoustic boundary conditions $s_{\mathrm{xx}}=s_{\mathrm{xx}}^{\mathrm{eff}}$ and $s_{\mathrm{yy}}=$ $s_{\text {yy }}^{\text {eff }}$ from (15), we have that

$$
\left.\frac{\partial s_{\mathrm{xx}}}{\partial s_{\mathrm{xx}}^{\mathrm{eff}}}\right|_{s_{\mathrm{yy}}^{\mathrm{eff}}, s_{\mathrm{zz}}^{\mathrm{eff}}}=1,\left.\quad \frac{\partial s_{\mathrm{yy}}}{\partial s_{\mathrm{xx}}^{\mathrm{eff}}}\right|_{s_{\mathrm{yy}}^{\mathrm{eff}}, s_{\mathrm{zz}}^{\mathrm{eff}}}=0,
$$

respectively. The boundary condition $\sigma_{\mathrm{zz}}=\sigma_{\mathrm{zz}}^{\text {eff }}$ and the constitutive relations for the constituent layers (16) give

$$
\begin{aligned}
& C_{\mathrm{xxzz}}^{\mathrm{eff}} s_{\mathrm{xx}}^{\mathrm{eff}}+C_{\mathrm{xxzz}}^{\mathrm{eff}} s_{\mathrm{yy}}^{\mathrm{eff}}+C_{\mathrm{zzzz}}^{\mathrm{eff}} s_{\mathrm{zz}}^{\mathrm{eff}} \\
& =C_{\mathrm{xxyy}} s_{\mathrm{xx}}+C_{\mathrm{xxyy}} s_{\mathrm{yy}}+C_{\mathrm{xxxx}} s_{\mathrm{zz}},
\end{aligned}
$$

which after implicit differentiation, where we also hold the strain fields $s_{\mathrm{yy}}^{\text {eff }}$ and $s_{\mathrm{zz}}^{\text {eff }}$ constant, takes the form

$$
\begin{gathered}
\left.C_{\mathrm{xxzz}}^{\mathrm{eff}} \frac{\partial s_{\mathrm{xx}}^{\mathrm{eff}}}{\partial s_{\mathrm{xx}}^{\mathrm{eff}}}\right|_{s_{\mathrm{yy}}^{\mathrm{eff}}, s_{\mathrm{zz}}^{\mathrm{eff}}}+\left.C_{\mathrm{xxzz}}^{\mathrm{eff}} \frac{\partial s_{\mathrm{yy}}^{\mathrm{eff}}}{\partial s_{\mathrm{xx}}^{\mathrm{eff}}}\right|_{s_{\mathrm{yy}}^{\mathrm{eff}}, s_{\mathrm{zz}}^{\mathrm{eff}}}+\left.C_{\mathrm{zzzz}}^{\mathrm{eff}} \frac{\partial s_{\mathrm{zz}}^{\mathrm{eff}}}{\partial s_{\mathrm{xx}}^{\mathrm{eff}}}\right|_{s_{\mathrm{yy}}^{\mathrm{eff}}, s_{\mathrm{zz}}^{\mathrm{eff}}}+\left.C_{\mathrm{xxyy}} \frac{\partial s_{\mathrm{xx}}}{\partial s_{\mathrm{xx}}^{\mathrm{eff}}}\right|_{s_{\mathrm{yy}}^{\mathrm{eff}}, s_{\mathrm{zz}}^{\mathrm{eff}}}+\left.C_{\mathrm{xxyy}} \frac{\partial s_{\mathrm{yy}}}{\partial s_{\mathrm{xx}}^{\mathrm{eff}}}\right|_{s_{\mathrm{yy}}^{\mathrm{eff}}, s_{\mathrm{zz}}^{\mathrm{eff}}}+\left.C_{\mathrm{xxxx}} \frac{\partial s_{\mathrm{zz}}}{\partial s_{\mathrm{xx}}^{\mathrm{eff}}}\right|_{s_{\mathrm{yy}}^{\mathrm{eff}}, s_{\mathrm{zz}}} .
\end{gathered}
$$


The boundary conditions in (15) and constant field requirements evident from (20a) reduce (26a) to the form

$$
C_{\mathrm{xxzz}}^{\mathrm{eff}}=C_{\mathrm{xxyy}}+\left.C_{\mathrm{xxxx}} \frac{\partial s_{\mathrm{zz}}}{\partial s_{\mathrm{xx}}^{\mathrm{eff}}}\right|_{s_{\mathrm{yy}}^{\text {eff }}, s_{\mathrm{zz}}^{\text {eff }}}
$$

and ultimately admits

$$
\left.\frac{\partial s_{\mathrm{zz}}}{\partial s_{\mathrm{xx}}^{\mathrm{eff}}}\right|_{s_{\mathrm{yy}}^{\mathrm{eff}}, s_{\mathrm{zz}}^{\mathrm{eff}}}=\frac{C_{\mathrm{xxzz}}^{\mathrm{eff}}-C_{\mathrm{xxzz}}}{C_{\mathrm{xxxx}}} .
$$

Substituting (24) and (26c) into the derivative (23) gives

$$
\left.\frac{\partial \varepsilon_{\mathrm{zz}}}{\partial s_{\mathrm{xx}}^{\mathrm{eff}}}\right|_{s_{\mathrm{yy}}^{\mathrm{eff}}, s_{\mathrm{zz}}^{\mathrm{eff}}}=-\left(\varepsilon_{\mathrm{zz}}\right)^{2} p_{\mathrm{xxzz}}-\left(\varepsilon_{\mathrm{zz}}\right)^{2} p_{\mathrm{xxxx}}\left[\frac{C_{\mathrm{xxzz}}^{\mathrm{eff}}-C_{\mathrm{xxzz}}}{C_{\mathrm{xxxx}}}\right],
$$

after using (21a), and analogously, we have that

$$
\left.\frac{\partial \varepsilon_{\mathrm{zz}}^{\prime}}{\partial s_{\mathrm{xx}}^{\mathrm{eff}}}\right|_{s_{\mathrm{yy}}^{\mathrm{eff}}, s_{\mathrm{zz}}^{\mathrm{eff}}}=-\left(\varepsilon_{\mathrm{zz}}^{\prime}\right)^{2} p_{\mathrm{xxzz}}^{\prime}-\left(\varepsilon_{\mathrm{zz}}^{\prime}\right)^{2} p_{\mathrm{xxxx}}^{\prime}\left[\frac{C_{\mathrm{xxzz}}^{\mathrm{eff}}-C_{\mathrm{xxzz}}^{\prime}}{C_{\mathrm{xxxx}}^{\prime}}\right] \text {, }
$$

for the second optically isotropic layer, following the boundary condition $\sigma_{\mathrm{zz}}^{\prime}=\sigma_{\mathrm{zz}}^{\mathrm{eff}}(15)$.

The derivative of the filling fraction in (22), through an application of the chain rule, gives rise to

$$
\begin{aligned}
& \left.\frac{\partial f}{\partial s_{\mathrm{xx}}^{\text {eff }}}\right|_{s_{\mathrm{yy}}^{\text {eff }}, s_{\mathrm{zz}}^{\text {eff }}}=\left.\left.a \frac{\partial f}{\partial a}\right|_{s_{\mathrm{yy}}^{\text {eff }}, s_{\mathrm{zz}}^{\text {eff }}, a^{\prime}} \frac{\partial s_{\mathrm{zz}}}{\partial s_{\mathrm{xx}}^{\mathrm{eff}}}\right|_{s_{\mathrm{yy}}^{\text {eff }}, s_{\mathrm{zz}}^{\text {eff }}} \\
& +\left.\left.a^{\prime} \frac{\partial f}{\partial a^{\prime}}\right|_{s_{\mathrm{yy}}^{\mathrm{eff}}, s_{\mathrm{zz}}^{\text {eff }}, a} \frac{\partial s_{\mathrm{zz}}^{\prime}}{\partial s_{\mathrm{xx}}^{\mathrm{eff}}}\right|_{s_{\mathrm{yy}}^{\mathrm{eff}}, s_{\mathrm{zz}}^{\mathrm{eff}}},
\end{aligned}
$$

following from the definitions $f=a /\left(a+a^{\prime}\right), \Delta s_{\mathrm{zz}}=\Delta a / a$, and $\Delta s_{\mathrm{zz}}^{\prime}=\Delta a^{\prime} / a^{\prime}$. Using (26c) and the analogous expression for the second layer, we obtain

$$
\left.\frac{\partial f}{\partial s_{\mathrm{xx}}^{\mathrm{eff}}}\right|_{S_{\mathrm{yy}}^{\mathrm{eff}}, s_{\mathrm{zz}}^{\text {eff }}}=f(1-f)\left[\frac{C_{\mathrm{xxzz}}^{\mathrm{eff}}-C_{\mathrm{xxyy}}}{C_{\mathrm{xxxx}}}-\frac{C_{\mathrm{xxzz}}^{\mathrm{eff}}-C_{\mathrm{xxyy}}^{\prime}}{C_{\mathrm{xxxx}}^{\prime}}\right] .
$$

Substituting (20a), (27a), (27b), and (28b) into expression (22) gives

$$
\begin{aligned}
p_{\mathrm{zzxx}}^{\mathrm{eff}}= & f p_{\mathrm{xxyy}}+(1-f) p_{\mathrm{xxyy}}^{\prime} \\
& -\frac{f(1-f)\left(p_{\mathrm{xxxx}}-p_{\mathrm{xxxx}}^{\prime}\right)\left(C_{\mathrm{xxyy}}-C_{\mathrm{xxyy}}^{\prime}\right)}{f C_{\mathrm{xxxx}}^{\prime}+(1-f) C_{\mathrm{xxxx}}} \\
& -f(1-f)\left(\frac{1}{\varepsilon_{\mathrm{zz}}}-\frac{1}{\varepsilon_{\mathrm{zz}}^{\prime}}\right)\left(\frac{C_{\mathrm{xxyy}}-C_{\mathrm{xxyy}}^{\prime}}{f C_{\mathrm{xxxx}}^{\prime}+(1-f) C_{\mathrm{xxxx}}}\right) .
\end{aligned}
$$

Having determined the analytical expression for $p_{\mathrm{zzxx}}^{\text {eff }}$, we now turn to the derivation of the $p_{\mathrm{zzzz}}^{\text {eff }}$ coefficient. Similarly, implicit differentiation of the effective permittivity expression (9c) with respect to $s_{\mathrm{zz}}^{\text {eff }}$, with $s_{\mathrm{xx}}^{\text {eff }}$ and $s_{\mathrm{yy}}^{\text {eff }}$ held constant, admits

$$
\begin{aligned}
-\left.\frac{1}{\left(\varepsilon_{\mathrm{zz}}^{\mathrm{eff}}\right)^{2}} \frac{\partial \varepsilon_{\mathrm{zz}}^{\mathrm{eff}}}{\partial s_{\mathrm{zz}}^{\mathrm{eff}}}\right|_{s_{\mathrm{xx}}^{\mathrm{eff}}, s_{\mathrm{yy}}^{\mathrm{eff}}}= & -\left.\frac{f}{\left(\varepsilon_{\mathrm{zz}}\right)^{2}} \frac{\partial \varepsilon_{\mathrm{zz}}}{\partial s_{\mathrm{zz}}^{\mathrm{eff}}}\right|_{s_{\mathrm{xx}}^{\mathrm{eff}}, s_{\mathrm{yy}}}-\left.\frac{(1-f)}{\left(\varepsilon_{\mathrm{zz}}^{\prime}\right)^{2}} \frac{\partial \varepsilon_{\mathrm{zz}}^{\prime}}{\partial s_{\mathrm{zz}}^{\mathrm{eff}}}\right|_{s_{\mathrm{xx}}^{\mathrm{eff}}, s_{\mathrm{yy}}^{\mathrm{eff}}} \\
& +\left.\left(\frac{1}{\varepsilon_{\mathrm{zz}}}-\frac{1}{\varepsilon_{\mathrm{zz}}^{\prime}}\right) \frac{\partial f}{\partial s_{\mathrm{zz}}^{\mathrm{eff}}}\right|_{s_{\mathrm{xx}}^{\mathrm{eff}}, s_{\mathrm{yy}}^{\mathrm{eff}}},
\end{aligned}
$$

where the first derivative is given by (20c). In a procedure analogous to above, we have that

$$
\begin{aligned}
\left.\frac{\partial \varepsilon_{\mathrm{zz}}}{\partial s_{\mathrm{zz}}^{\mathrm{eff}}}\right|_{s_{\mathrm{xx}}^{\text {eff }}, s_{\mathrm{yy}}^{\text {eff }}}= & \left.\left.\frac{\partial \varepsilon_{\mathrm{zz}}}{\partial s_{\mathrm{xx}}}\right|_{s_{\mathrm{xx}}^{\text {eff }}, s_{\mathrm{yy}}^{\text {eff }}} \frac{\partial s_{\mathrm{xx}}}{\partial s_{\mathrm{zz}}^{\mathrm{eff}}}\right|_{s_{\mathrm{xx}}^{\text {eff }}, s_{\mathrm{yy}}^{\text {eff }}}+\left.\left.\frac{\partial \varepsilon_{\mathrm{zz}}}{\partial s_{\mathrm{yy}}}\right|_{s_{\mathrm{xx}}^{\text {eff }}, s_{\mathrm{yy}} \text { eff }} \frac{\partial s_{\mathrm{yy}}}{\partial s_{\mathrm{zz}}^{\text {eff }}}\right|_{s_{\mathrm{xx}}^{\text {eff }}, s_{\mathrm{yy}}^{\text {eff }}} \\
& +\left.\left.\frac{\partial \varepsilon_{\mathrm{zz}}}{\partial s_{\mathrm{zz}}}\right|_{s_{\mathrm{xx}}^{\text {eff }}, s_{\mathrm{yy}}^{\text {eff }}} \frac{\partial s_{\mathrm{zz}}}{\partial s_{\mathrm{zz}}^{\text {eff }}}\right|_{s_{\mathrm{xx}}^{\text {eff }}, s_{\mathrm{yy}}^{\text {eff }}},
\end{aligned}
$$

where the boundary conditions (15) and constant-field requirements give rise to

$$
\left.\frac{\partial s_{\mathrm{xx}}}{\partial s_{\mathrm{zz}}^{\text {eff }}}\right|_{s_{\mathrm{xx}}^{\text {eff }}, s_{\mathrm{yy}}^{\text {eff }}}=\left.\frac{\partial s_{\mathrm{yy}}}{\partial s_{\mathrm{zZ}}^{\text {eff }}}\right|_{s_{\mathrm{yy}}^{\text {eff }}, s_{\mathrm{zz}}^{\text {eff }}}=0 .
$$

The remaining boundary condition $\sigma_{\mathrm{zz}}=\sigma_{\mathrm{zz}}^{\text {eff }}$ and constitutive relations (16) give the expression (25) once more. Implicit differentiation with respect to $s_{\mathrm{zz}}^{\mathrm{eff}}$ and the new constant-field requirements admits

$$
\begin{aligned}
& \left.C_{\mathrm{xxzz}}^{\mathrm{eff}} \frac{\partial s_{\mathrm{xx}}^{\mathrm{eff}}}{\partial s_{\mathrm{zz}}^{\mathrm{eff}}}\right|_{s_{\mathrm{xx}}^{\mathrm{eff}}, s_{\mathrm{yy}}^{\text {eff }}}+\left.C_{\mathrm{xxzz}}^{\mathrm{eff}} \frac{\partial s_{\mathrm{yy}}^{\mathrm{eff}}}{\partial s_{\mathrm{zz}}^{\mathrm{eff}}}\right|_{s_{\mathrm{xx}}^{\text {eff }}, s_{\mathrm{yy}}^{\mathrm{eff}}}+\left.C_{\mathrm{zzzz}}^{\mathrm{eff}} \frac{\partial s_{\mathrm{zz}}^{\mathrm{eff}}}{\partial s_{\mathrm{zz}}^{\mathrm{eff}}}\right|_{s_{\mathrm{xx}}^{\mathrm{eff}}, s_{\mathrm{yy}}^{\text {eff }}} \\
& =\left.C_{\mathrm{xxyy}} \frac{\partial s_{\mathrm{xx}}}{\partial s_{\mathrm{zz}}^{\mathrm{eff}}}\right|_{s_{\mathrm{xx}}^{\mathrm{eff}},,_{\mathrm{yy}}^{\mathrm{eff}}}+\left.C_{\mathrm{xxyy}} \frac{\partial s_{\mathrm{yy}}}{\partial s_{\mathrm{zz}}^{\mathrm{eff}}}\right|_{s_{\mathrm{xx}}^{\mathrm{eff}}, s_{\mathrm{yy}}^{\mathrm{eff}}}+\left.C_{\mathrm{xxxx}} \frac{\partial s_{\mathrm{zz}}}{\partial s_{\mathrm{zz}}^{\mathrm{eff}}}\right|_{s_{\mathrm{xx}}^{\mathrm{eff}},,_{\mathrm{yy}}^{\mathrm{eff}}} \text {, }
\end{aligned}
$$

and ultimately, we find that

$$
\left.\frac{\partial s_{\mathrm{zz}}}{\partial s_{\mathrm{zZ}}^{\mathrm{eff}}}\right|_{s_{\mathrm{xx}}^{\mathrm{eff}}, s_{\mathrm{yy}}^{\mathrm{eff}}}=\frac{C_{\mathrm{zzzz}}^{\mathrm{eff}}}{C_{\mathrm{xxxx}}} .
$$

Subsequently, substituting (32) and (34) into (31), we obtain

$$
\left.\frac{\partial \varepsilon_{\mathrm{zz}}}{\partial s_{\mathrm{zz}}^{\mathrm{eff}}}\right|_{s_{\mathrm{xx}}^{\mathrm{eff}}, s_{\mathrm{yy}}^{\mathrm{eff}}}=-\left(\varepsilon_{\mathrm{zz}}\right)^{2} p_{\mathrm{xxxx}}\left(\frac{C_{\mathrm{zzzz}}^{\mathrm{eff}}}{C_{\mathrm{xxxx}}}\right),
$$

after using (21a), and analogously, we have

$$
\left.\frac{\partial \varepsilon_{\mathrm{zz}}^{\prime}}{\partial s_{\mathrm{zz}}^{\mathrm{eff}}}\right|_{S_{\mathrm{xx}}^{\mathrm{eff}}, s_{\mathrm{yy}}^{\mathrm{eff}}}=-\left(\varepsilon_{\mathrm{zz}}^{\prime}\right)^{2} p_{\mathrm{xxxx}}^{\prime}\left(\frac{C_{\mathrm{zzzz}}^{\mathrm{eff}}}{C_{\mathrm{xxxx}}^{\prime}}\right) .
$$

The derivative of the filling fraction in (30) takes the form

$$
\begin{aligned}
\left.\frac{\partial f}{\partial s_{\mathrm{zz}}^{\mathrm{eff}}}\right|_{s_{\mathrm{xx}}^{\mathrm{eff}}, s_{\mathrm{yy}}^{\mathrm{eff}}} & =f(1-f)\left[\left.\frac{\partial s_{\mathrm{zz}}}{\partial s_{\mathrm{zz}}^{\mathrm{eff}}}\right|_{s_{\mathrm{xx}}^{\mathrm{eff}}, s_{\mathrm{yy}}^{\mathrm{eff}}}-\left.\frac{\partial s_{\mathrm{zz}}^{\prime}}{\partial s_{\mathrm{zz}}^{\mathrm{eff}}}\right|_{s_{\mathrm{xx}}^{\mathrm{eff}}, s_{\mathrm{yy}}^{\mathrm{eff}}}\right] \\
& =f(1-f)\left[\frac{C_{\mathrm{zzzz}}^{\mathrm{eff}}}{C_{\mathrm{xxxx}}}-\frac{C_{\mathrm{zzzz}}^{\mathrm{eff}}}{C_{\mathrm{xxxx}}^{\prime}}\right],
\end{aligned}
$$

following (34) and the corresponding expression for the second layer. Substituting (35a), (35b), and (36) into (30), we obtain

$$
\begin{aligned}
\frac{p_{\mathrm{zzzz}}^{\mathrm{eff}}}{C_{\mathrm{zzzz}}^{\mathrm{eff}}}= & f\left(\frac{p_{\mathrm{xxxx}}}{C_{\mathrm{xxxx}}}\right)+(1-f)\left(\frac{p_{\mathrm{xxxx}}^{\prime}}{C_{\mathrm{xxxx}}^{\prime}}\right) \\
& +f(1-f)\left(\frac{1}{\varepsilon_{\mathrm{zz}}}-\frac{1}{\varepsilon_{\mathrm{zz}}^{\prime}}\right)\left(\frac{1}{C_{\mathrm{xxxx}}}-\frac{1}{C_{\mathrm{xxxx}}^{\prime}}\right) .
\end{aligned}
$$

The expressions for $p_{\mathrm{zzxx}}^{\text {eff }}$ in (29) and $p_{\mathrm{zzzz}}^{\mathrm{eff}}$ in (37) are presented below along with all other remaining 
coefficients:

$$
\begin{aligned}
& \left(\varepsilon_{\mathrm{xx}}^{\mathrm{eff}}\right)^{2} p_{\mathrm{xxxx}}^{\mathrm{eff}}=f\left(\varepsilon_{\mathrm{xx}}\right)^{2} p_{\mathrm{xxxx}}+(1-f)\left(\varepsilon_{\mathrm{xx}}^{\prime}\right)^{2} p_{\mathrm{xxxx}}^{\prime} \\
& -\frac{f(1-f)\left(C_{\mathrm{xxyy}}-C_{\mathrm{xxyy}}^{\prime}\right)\left[\left(\varepsilon_{\mathrm{xx}}\right)^{2} p_{\mathrm{xxyy}}-\left(\varepsilon_{\mathrm{xx}}^{\prime}\right)^{2} p_{\mathrm{xxyy}}^{\prime}\right]}{f C_{\mathrm{xxxx}}^{\prime}+(1-f) C_{\mathrm{xxxx}}}+\frac{f(1-f)\left(\varepsilon_{\mathrm{xx}}-\varepsilon_{\mathrm{xx}}^{\prime}\right)\left(C_{\mathrm{xxyy}}-C_{\mathrm{xxyy}}^{\prime}\right)}{f C_{\mathrm{xxxx}}^{\prime}+(1-f) C_{\mathrm{xxxx}}}, \\
& \frac{p_{\mathrm{zzzz}}^{\mathrm{eff}}}{C_{\mathrm{zzzz}}^{\mathrm{eff}}}=f\left(\frac{p_{\mathrm{xxxx}}}{C_{\mathrm{xxxx}}}\right)+(1-f)\left(\frac{p_{\mathrm{xxxx}}^{\prime}}{C_{\mathrm{xxxx}}^{\prime}}\right)+f(1-f)\left(\frac{1}{\varepsilon_{\mathrm{zz}}}-\frac{1}{\varepsilon_{\mathrm{zz}}^{\prime}}\right)\left(\frac{1}{C_{\mathrm{xxxx}}}-\frac{1}{C_{\mathrm{xxxx}}^{\prime}}\right), \\
& \frac{\left(\varepsilon_{\mathrm{xx}}^{\mathrm{eff}}\right)^{2} p_{\mathrm{xxzz}}^{\mathrm{eff}}}{C_{\mathrm{zzzz}}^{\mathrm{eff}}}=f \frac{\left(\varepsilon_{\mathrm{xx}}\right)^{2} p_{\mathrm{xxyy}}}{C_{\mathrm{xxxx}}}+(1-f) \frac{\left(\varepsilon_{\mathrm{xx}}^{\prime}\right)^{2} p_{\mathrm{xxyy}}^{\prime}}{C_{\mathrm{xxxx}}^{\prime}}-f(1-f)\left(\varepsilon_{\mathrm{xx}}-\varepsilon_{\mathrm{xx}}^{\prime}\right)\left(\frac{1}{C_{\mathrm{xxxx}}}-\frac{1}{C_{\mathrm{xxxx}}^{\prime}}\right), \\
& \left(\varepsilon_{\mathrm{xx}}^{\mathrm{eff}}\right)^{2} p_{\mathrm{xxyy}}^{\mathrm{eff}}=f\left(\varepsilon_{\mathrm{xx}}\right)^{2} p_{\mathrm{xxyy}}+(1-f)\left(\varepsilon_{\mathrm{xx}}^{\prime}\right)^{2} p_{\mathrm{xxyy}}^{\prime} \\
& -\frac{f(1-f)\left(C_{\mathrm{xxyy}}-C_{\mathrm{xxyy}}^{\prime}\right)\left[\left(\varepsilon_{\mathrm{xx}}\right)^{2} p_{\mathrm{xxyy}}-\left(\varepsilon_{\mathrm{xx}}^{\prime}\right)^{2} p_{\mathrm{xxyy}}^{\prime}\right]}{f C_{\mathrm{xxxx}}^{\prime}+(1-f) C_{\mathrm{xxxx}}}+\frac{f(1-f)\left(\varepsilon_{\mathrm{xx}}-\varepsilon_{\mathrm{xx}}^{\prime}\right)\left(C_{\mathrm{xxyy}}-C_{\mathrm{xxyy}}^{\prime}\right)}{f C_{\mathrm{xxxx}}^{\prime}+(1-f) C_{\mathrm{xxxx}}}, \\
& p_{\mathrm{zzxx}}^{\mathrm{eff}}=f p_{\mathrm{xxyy}}+(1-f) p_{\mathrm{xxyy}}^{\prime} \\
& -\frac{f(1-f)\left(p_{\mathrm{xxxx}}-p_{\mathrm{xxxx}}^{\prime}\right)\left(C_{\mathrm{xxyy}}-C_{\mathrm{xxyy}}^{\prime}\right)}{f C_{\mathrm{xxxx}}^{\prime}+(1-f) C_{\mathrm{xxxx}}}-f(1-f)\left(\frac{1}{\varepsilon_{\mathrm{zz}}}-\frac{1}{\varepsilon_{\mathrm{zz}}^{\prime}}\right)\left(\frac{C_{\mathrm{xxyy}}-C_{\mathrm{xxyy}}^{\prime}}{f C_{\mathrm{xxxx}}^{\prime}+(1-f) C_{\mathrm{xxxx}}}\right), \\
& \frac{\varepsilon_{\mathrm{yy}}^{\mathrm{eff}} p_{\mathrm{yzyz}}^{\mathrm{eff}}}{C_{\mathrm{yzyz}}^{\mathrm{eff}}}=f \frac{\varepsilon_{\mathrm{yy}} p_{\mathrm{yzyz}}}{C_{\mathrm{yzyz}}}+(1-f) \frac{\varepsilon_{\mathrm{yy}}^{\prime} p_{\mathrm{yzyz}}^{\prime}}{C_{\mathrm{yzyz}}^{\prime}}, \\
& \varepsilon_{\mathrm{xx}}^{\mathrm{eff}} \varepsilon_{\mathrm{yy}}^{\mathrm{eff}} p_{\mathrm{xyxy}}^{\mathrm{eff}}=f \varepsilon_{\mathrm{xx}} \varepsilon_{\mathrm{yy}} p_{\mathrm{xyxy}}+(1-f) \varepsilon_{\mathrm{xx}}^{\prime} \varepsilon_{\mathrm{yy}}^{\prime} p_{\mathrm{xyxy}}^{\prime} .
\end{aligned}
$$

We remark that in the expressions above, the photoelastic coefficients possess the form $p_{i j k l}^{\text {eff }}=\alpha_{\text {qrst }} p_{q r s t}+\alpha_{q r s t}^{\prime} p_{q r s t}^{\prime}+$ $p_{i j k l}^{\text {art }}$, where $\alpha_{q r s t}$ and $\alpha_{q r s t}^{\prime}$ are functions of material parameters but may be regarded as weightings for the photoelastic coefficients of the constituent layers. Following the convention established in earlier work [16], the final contribution $p_{i j k l}^{\text {art }}$ is termed artificial photoelasticity, as this represents a nontrivial contribution to the photoelastic properties of the composite when $p_{q r s t}=p_{q r s t}^{\prime}=0$. These artificial contributions are directly proportional to the contrast in relevant components of the permittivity and stiffness tensors and have been shown to play a significant role in the photoelastic properties of other subwavelength structured designs $[17,18]$. Note that for $p_{\text {yzyz }}^{\text {eff }}$ and $p_{\mathrm{xyxy}}^{\text {eff }}$ above, there is no artificial photoelastic component, as shear waves do not change the volume of the unit cell when the constituent and effective material are oriented with the Cartesian coordinate frame, i.e.,

$$
\frac{\partial f}{\partial s_{\mathrm{yz}}^{\text {eff }}}=\frac{\partial f}{\partial s_{\mathrm{xz}}^{\text {eff }}}=\frac{\partial f}{\partial s_{\mathrm{xy}}^{\text {eff }}}=0
$$

however, we emphasize that this result holds only for highsymmetry composites.

The derivation outlined in this section gives results for the symmetric photoelastic strain tensor; expressions for the symmetric photoelastic stress tensor may be found through a straightforward application of Hooke's law [21]. However, the photoelastic strain coefficients $p_{i j k l}^{\text {eff }}$ may be expressed in terms of both effective stress and strain fields; if chosen appropriately, the photoelastic coefficients are then derived in terms of acoustic fields that are everywhere continuous in the layered medium. Such an approach is analogous to that outlined in Rouhani and Sapriel [19]. For example, substituting one line of the constitutive relation (16) into the photoelastic tensor definition (19), we obtain

$$
\begin{aligned}
\Delta \varepsilon_{\mathrm{zz}}^{\mathrm{eff}}= & -\left(\varepsilon_{\mathrm{zz}}^{\mathrm{eff}}\right)^{2}\left[\left\{p_{\mathrm{zzxx}}^{\mathrm{eff}}-\frac{C_{\mathrm{xxzz}}^{\mathrm{eff}}}{C_{\mathrm{zzzz}}^{\mathrm{eff}}} p_{\mathrm{zzzz}}^{\mathrm{eff}}\right\} s_{\mathrm{xx}}^{\mathrm{eff}}\right. \\
& \left.+\left\{p_{\mathrm{zzxx}}^{\mathrm{eff}}-\frac{C_{\mathrm{xxzz}}^{\mathrm{eff}}}{C_{\mathrm{zzzz}}^{\mathrm{eff}}} p_{\mathrm{zzzz}}^{\mathrm{eff}}\right\} s_{\mathrm{yy}}^{\mathrm{eff}}+\frac{p_{\mathrm{zzzz}}^{\mathrm{eff}}}{C_{\mathrm{zzzz}}^{\mathrm{eff}}} \sigma_{\mathrm{zz}}^{\mathrm{eff}}\right],
\end{aligned}
$$

where photoelastic strain coefficients are now obtained through differentiation (as before), but with effective stress and effective strain fields held constant. However, such a procedure gives final expressions for $p_{i j k l}^{\text {eff }}$ identical to those presented in (38). As a final remark, the $p_{i j k l}$ coefficients presented in (38), with $p_{i j k l}^{\text {art }}=0$, are identical to those tabulated in Rouhani and Sapriel [19] after considering symmetry reductions of tensor coefficients [21].

\section{Effective antisymmetric photoelastic tensor}

In this section, we evaluate the antisymmetric component of the photoelastic tensor $r_{i j k l}^{\text {eff }}$ defined by

$$
\Delta\left(\varepsilon_{\mathrm{eff}}^{-1}\right)_{i j}=r_{i j k l}^{\mathrm{eff}} r_{k l}^{\mathrm{eff}},
$$

where $r_{k l}=\frac{1}{2}\left(\partial_{l} u_{k}-\partial_{k} u_{l}\right)$ denotes the infinitesimal rotation tensor. The derivation for the roto-optic tensor of a uniform material is given in $[3,20]$ and extends to the case of a subwavelength structured material as

$$
\begin{aligned}
r_{i j k l}^{\mathrm{eff}}= & \frac{1}{2}\left[\left(\varepsilon_{\mathrm{eff}}^{-1}\right)_{i l} \delta_{k j}+\left(\varepsilon_{\mathrm{eff}}^{-1}\right)_{l j} \delta_{i k}\right. \\
& \left.-\left(\varepsilon_{\mathrm{eff}}^{-1}\right)_{i k} \delta_{l j}-\left(\varepsilon_{\mathrm{eff}}^{-1}\right)_{k j} \delta_{i l}\right] .
\end{aligned}
$$




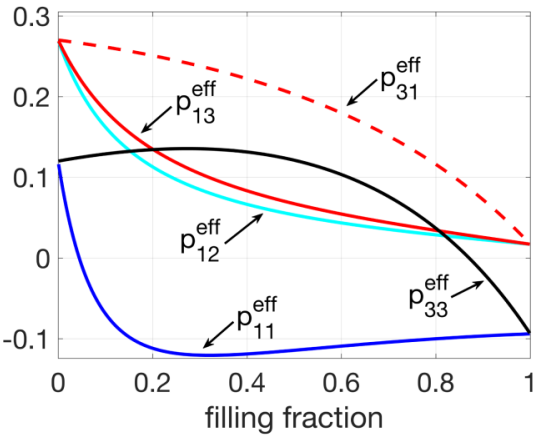

(a)

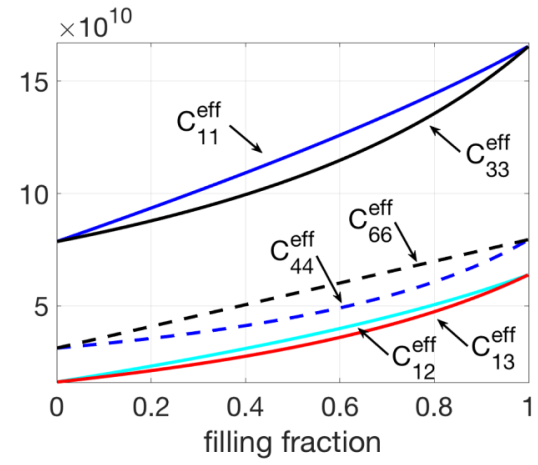

(d)

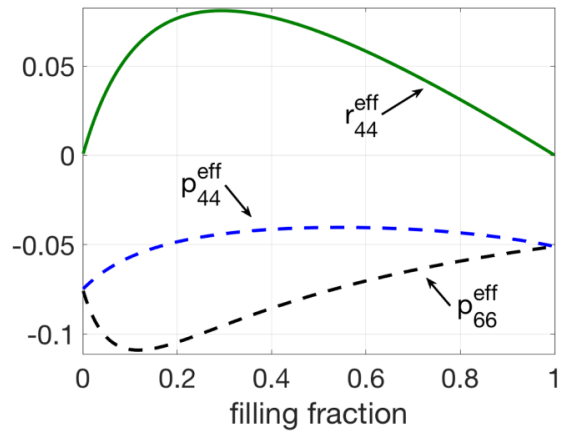

(b)

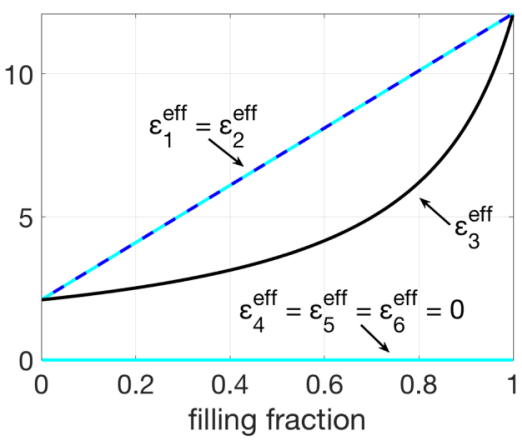

(e)

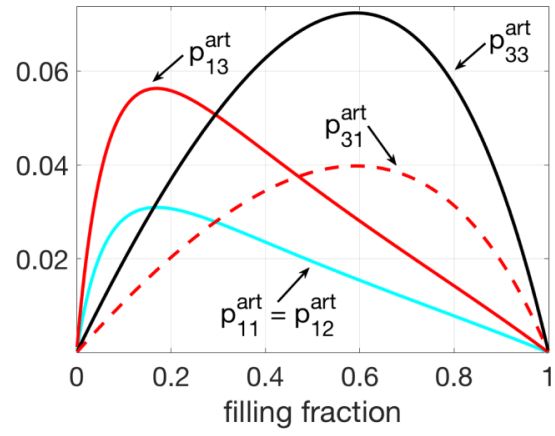

(c)

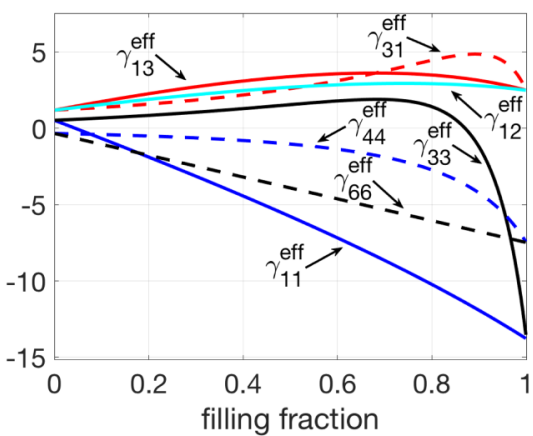

(f)

FIG. 2. (a) Symmetric photoelastic coefficients $p_{i j k l}^{\text {eff }}$ corresponding to simple strains, (b) roto-optic coefficient $r_{\text {yzyz }}^{\text {eff }}$ and symmetric photoelastic coefficients $p_{\mathrm{yzyz}}^{\text {eff }}$ and $p_{\mathrm{xyxy}}^{\text {eff }}$, (c) artificial photoelastic terms $p_{i j k l}^{\text {art }}$, (d) stiffness tensor coefficients $C_{i j k l}^{\text {eff }}$, (e) permittivity coefficients $\varepsilon_{i j}^{\text {eff }}$, and (f) symmetric electrostriction coefficients $\gamma_{i j k l}^{\text {eff }}$ as a function of filling fraction $f$ for silica and Si [100] layers with $a+a^{\prime}=50 \mathrm{~nm}$ and labels in Voigt notation.

This simplifies to the form [22]

$$
r_{i j k l}^{\mathrm{eff}}=\frac{1}{2}\left(\frac{1}{\varepsilon_{j j}^{\mathrm{eff}}}-\frac{1}{\varepsilon_{i i}^{\mathrm{eff}}}\right)\left(\delta_{i k} \delta_{j l}-\delta_{i l} \delta_{j k}\right),
$$

provided the layered material does not possess triclinic or monoclinic symmetry. For our tetragonal $(4 / \mathrm{mmm})$ layered structure, there are only eight nonvanishing $r_{i j k l}$ terms, which all take the same value modulo a sign change that arises from the antisymmetric nature of the tensor $r_{i j k l}=-r_{i j l k}$. From the expressions for the effective permittivity presented in Sec. II A, we have that

$r_{\mathrm{xzxz}}^{\mathrm{eff}}=\frac{1}{2}\left(\frac{f \varepsilon_{\mathrm{zz}}^{\prime}+(1-f) \varepsilon_{\mathrm{zz}}}{\varepsilon_{\mathrm{zz}} \varepsilon_{\mathrm{zz}}^{\prime}}-\frac{1}{f \varepsilon_{\mathrm{xx}}+(1-f) \varepsilon_{\mathrm{xx}}^{\prime}}\right)$

for layers of optically isotropic media.

\section{NUMERICAL EXAMPLES}

In this section, we present the effective permittivity, stiffness, and photoelastic tensors for a selection of material combinations, where constituent parameter values are taken from Table 1 of Smith et al. [17]. Here values are presented at a vacuum wavelength of $\lambda=1550 \mathrm{~nm}$ and for a total layer cell width of $a+a^{\prime}=50 \mathrm{~nm}$.

We begin by considering the material properties of a fused silica and silicon [100] layered medium in Fig. 2. In Fig. 2(a) we present the symmetric photoelastic coefficients $p_{\text {xxxx }}^{\text {eff }}$ (blue curve), $p_{\text {xxyy }}^{\text {eff }}\left(\right.$ cyan curve), $p_{\text {xxzz }}^{\text {eff }}$ (solid red curve), $p_{\mathrm{zzzz}}^{\text {eff }}$ (black curve), and $p_{\mathrm{zzxx}}^{\text {eff }}$ (dashed red curve) as a function of filling fraction. Here the coefficients exhibit a varied dependence on filling fraction, with enhancement in the $p_{\mathrm{xxxx}}^{\text {eff }}$ and $p_{\mathrm{zzzz}}^{\text {eff }}$ elements beyond either of the constituent values to $p_{\mathrm{zzzz}}^{\mathrm{eff}}=0.135$ at $f=0.275$ and $p_{\mathrm{xxxx}}^{\text {eff }}=-0.121$ at $f=0.32$. For reference, we describe such behavior as extraordinary enhancement (i.e., when a composite material possesses material values that are beyond the values for either of the constituents). Interestingly, the off-diagonal elements $p_{\mathrm{xxyy}}^{\text {eff }}, p_{\mathrm{xxzz}}^{\text {eff }}$, and $p_{\mathrm{zzxx}}^{\mathrm{eff}}$ do not demonstrate extraordinary enhancement for this material combination. We also have $p_{\mathrm{xxxx}}^{\text {eff }}=0$ at $f=0.045$ along with $p_{\mathrm{zzzz}}^{\text {eff }}=0$ at $f=0.87$, which implies that longitudinal acoustic waves traveling along $x$ at $f=0.045$ and longitudinal acoustic waves traveling along $z$ at $f=0.87$ will not alter the optical properties of the medium. Reassuringly, symmetry-required degeneracies are recovered at $f=0$ and $f=1$, where the layered medium returns to a uniform material (i.e., $p_{\mathrm{xxxx}}=p_{\mathrm{zzzz}}$ and $p_{\mathrm{xxyy}}=$ $p_{\mathrm{xxzz}}=p_{\mathrm{zzxx}}$ in a cubic or isotropic medium). In Fig. 2(b) we show the remaining symmetric photoelastic coefficients $p_{\text {yzyz }}^{\text {eff }}$ and $p_{\text {xyxy }}^{\text {eff }}$ as functions of filling fraction, in addition to the roto-optic tensor coefficient $r_{\mathrm{xyxy}}^{\text {eff }}$. Here we observe a strong roto-optic effect in the layered material, which reaches a maximum of $r_{\mathrm{xyxy}}^{\text {eff }}=0.081$ at $f=0.295$. This value is greater than $\left|p_{\text {xyxy }}^{\mathrm{Si}}\right|=0.051$ and $\left|p_{\text {xyxy }}^{\mathrm{SiO}_{2}}\right|=0.075$ and is also different in sign, which demonstrates that the roto-optic effect can significantly alter the predicted change in permittivity for acoustic shear waves and should not be omitted a priori 


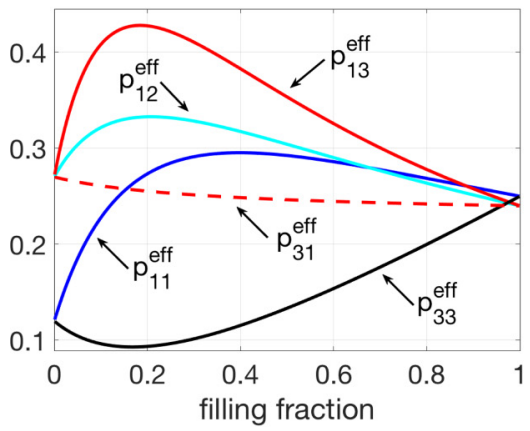

(a)

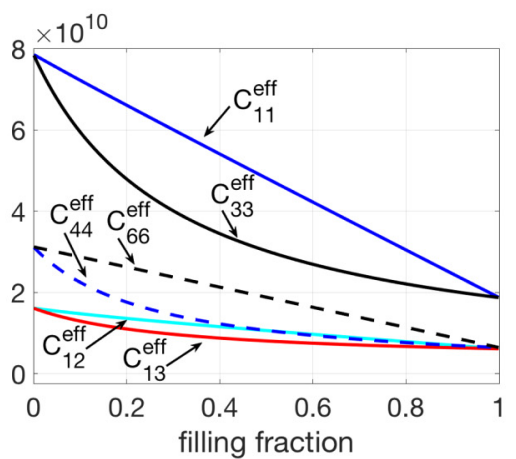

(d)

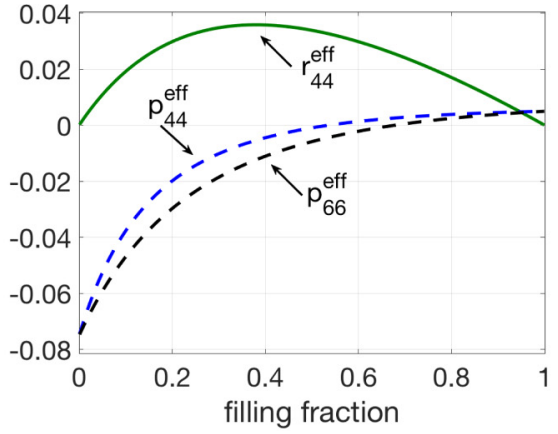

(b)

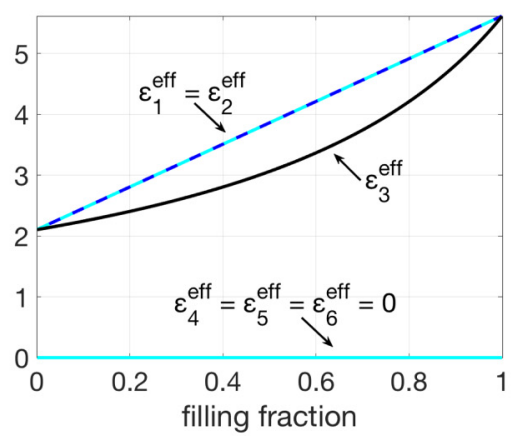

(e)

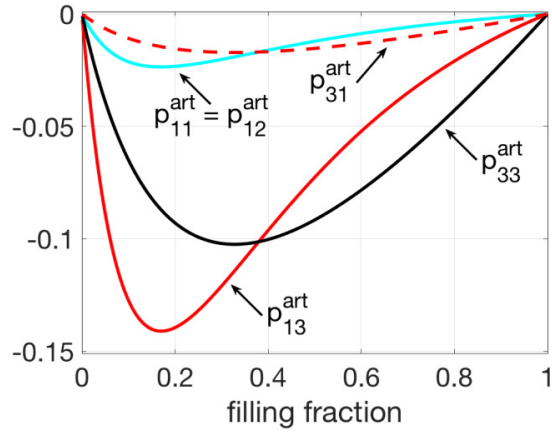

(c)

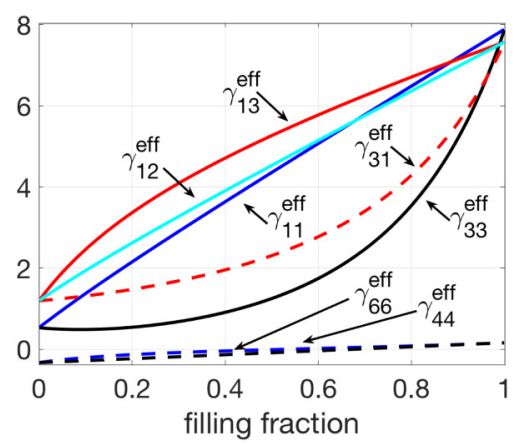

(f)

FIG. 3. (a) Symmetric photoelastic coefficients $p_{i j k l}^{\text {eff }}$ corresponding to simple strains, (b) roto-optic coefficient $r_{\text {yzyz }}^{\text {eff }}$ and symmetric photoelastic coefficients $p_{\text {yzyz }}^{\text {eff }}$ and $p_{\text {xyxy }}^{\text {eff }}$, (c) artificial photoelastic terms $p_{i j k l}^{\text {art }}$, (d) stiffness tensor coefficients $C_{i j k l}^{\text {eff }}$, (e) permittivity coefficients $\varepsilon_{i j}^{\text {eff }}$, and (f) symmetric electrostriction coefficients $\gamma_{i j k l}^{\text {eff }}$ as a function of filling fraction $f$ for silica and $\mathrm{As}_{2} \mathrm{~S}_{3}$ glass layers with $a+a^{\prime}=50 \mathrm{~nm}$ and labels in Voigt notation.

without careful consideration. The two photoelastic shear constants also exhibit extraordinary enhancement, taking extremal values of $p_{\text {yzyz }}^{\text {eff }}=-0.04$ at $f=0.545$ and $p_{\text {xyxy }}^{\text {eff }}=-0.109$ at $f=0.117$, and possess the correct degeneracy at $f=0$ and $f=1$ (i.e., $p_{\mathrm{yzyz}}=p_{\mathrm{xyxy}}$ in a cubic or isotropic medium).

In Fig. 2(c) we present the artificial contributions to the photoelastic tensors shown in Fig. 2(a), which all exhibit significant, positive-valued contributions to the effective symmetric photoelastic coefficients. This artificial contribution [obtained by substituting $p_{i j k l}=p_{i j k l}^{\prime}=0$ in (38)] diminishes the extreme range of $p_{\mathrm{xxxx}}^{\mathrm{eff}}$ and shows that the individual weightings for the constituent coefficients can take values $\left|\alpha_{\text {qrst }}\right|,\left|\alpha_{\text {qrst }}^{\prime}\right|>1$. This demonstrates that extraordinary enhancement is possible without artificial photoelasticity. In the case of a layered medium, we remark that $p_{\mathrm{xxxx}}^{\text {art }}=p_{\mathrm{xxyy}}^{\text {art }}$ since these terms both arise from in-plane strains and are related to the same in-plane permittivity and stiffness contrast. For reference, maximum values are listed as follows: $p_{\mathrm{xxxx}}^{\text {art }}=$ $p_{\text {xxyy }}^{\text {art }}=0.031$ at $f=0.17, p_{\mathrm{zzzz}}^{\text {art }}=0.072$ and $p_{\mathrm{zzxx}}^{\text {art }}=0.04$ at $f=0.592$, and $p_{\mathrm{xxzz}}^{\text {art }}=0.056$ at $f=0.17$. In Figs. 2(d) and 2(e), we present the effective stiffness and permittivity coefficients as functions of filling fraction, following their explicit definitions in (9) and (17). Here the simple dependences on $f$ are visible, extraordinary enhancements are not observed, and the required material symmetries are recovered at $f=0$ and $f=1$. In effect, for the permittivity and stiffness tensors, all in-plane terms are given by volume averaging, and all out-of-plane terms are given by the inverse of volume-averaged reciprocal values.
In Fig. 2(f) we give the corresponding symmetric electrostriction coefficients, defined as $\gamma_{i j k l}=\varepsilon_{i i} \varepsilon_{j j} p_{i j k l}$. In a manner analogous to that for the photoelastic coefficients, the electrostrictive coefficients of a composite material also exhibit a nontrivial dependence on $f$, in addition to extraordinary enhancement. Maxima of $\gamma_{\text {xxyy }}^{\text {eff }}=2.96$ at $f=0.74, \gamma_{\text {xxzz }}^{\text {eff }}=$ 3.626 at $f=0.68$, and $\gamma_{\mathrm{zzx}}^{\text {eff }}=4.87$ at $f=0.89$ are observed, demonstrating that the choice of polarization and propagation direction can have important implications for SBS experiments in layered media.

In Fig. 3 we present the material properties for a layered medium comprising fused silica and $\mathrm{As}_{2} \mathrm{~S}_{3}$ glass layers, in a manner analogous to Fig. 2. In Fig. 3(a) we show a selection of symmetric photoelastic constants for the composite, where it is observed that all $p_{i j k l}^{\text {eff }}$ corresponding to simple strains exhibit extraordinary enhancement. The enhancement of the $p_{\text {xxzz }}^{\text {eff }}$ coefficient to $p_{\text {xxzz }}^{\text {eff }}=0.428$ at $f=0.184$ is remarkable when compared to $p_{\mathrm{xxyy}}^{\mathrm{SiO}_{2}}=0.27$ and $p_{\mathrm{xxyy}}^{\mathrm{As}_{2} \mathrm{~S}_{3}}=0.24$ (i.e., an enhancement of $59 \%$ and $78 \%$, respectively). In Fig. 3(b) we show the dependence on filling fraction for the remaining symmetric photoelastic constants, in addition to the rotooptic coefficient. For silica and chalcogenide glass layers, a maximum of $r_{\text {yzyz }}^{\text {eff }}=0.036$ is achieved at $f=0.38$. Here we observe $p_{\text {yzyz }}^{\text {eff }}=0$ at $f=0.535$ and $p_{\text {xyxy }}^{\text {eff }}=0$ at $f=0.678$, revealing that only roto-optic contributions participate in acousto-optic interactions at these filling fractions.

In Fig. 3(c) we present the artificial contribution to the total symmetric photoelastic coefficients shown in Fig. 3(a). Here it is apparent that artificial terms contribute negatively to the 
photoelastic properties of the layered medium, reducing the $p_{\mathrm{xxzz}}^{\text {eff }}$ and $p_{\mathrm{zzzz}}^{\text {eff }}$ coefficients significantly. From this figure, we also determine that the extraordinary enhancement in $p_{\mathrm{xxzz}}^{\text {eff }}$ is due to $\left|\alpha_{\text {qrst }}\right|,\left|\alpha_{\text {qrst }}^{\prime}\right|>1$. For reference, a maximum value of $p_{\mathrm{xxzz}}^{\text {art }}=-0.141$ is achieved at $f=0.17$. In Fig. 3(d) we present the stiffness tensor coefficients for the layered medium, and in Fig. 3(e) we show the permittivity tensor coefficients as functions of filling fraction. Both of these figures exhibit a behavior qualitatively similar to that in Figs. 2(d) and 2(e) with the absence of extraordinary enhancement. In Fig. 3(f) we present the electrostriction constants as a function of the filling fraction for this material combination for completeness. Despite the large value for $p_{\text {xxzz }}^{\text {eff }}$ observed in Fig. 3(a), the corresponding $\gamma_{\mathrm{xxzz}}^{\text {eff }}$ term is smoothed by the much stronger growth in $\left(\varepsilon_{\mathrm{xx}}^{\mathrm{eff}}\right)^{2}$. Also, we observe zero values for $\gamma_{\mathrm{yzyz}}^{\text {eff }}$ and $\gamma_{\mathrm{xyxy}}^{\text {eff }}$ following Fig. 3(b) along with $\gamma_{\mathrm{xxxx}}^{\text {eff }}$ and $\gamma_{\mathrm{zzzz}}^{\text {eff }}$ following Fig. 3(a).

Following earlier works by some of the authors $[17,18]$ on the numerical study of photoelasticity in composites comprising arrays of spheres, we now briefly compare results for a layered structure of silicon and chalcogenide glass with a corresponding cubic lattice structure. The numerical procedure for the sphere configuration determines the effective bulk photoelastic response (including artificial contributions) by comparing the change in the effective permittivity tensor relative to a small mechanical strain imposed on the unit-cell boundary.

In Fig. 4 we compare the photoelastic constants obtained with silicon and chalcogenide glass as a function of filling fraction when they are structured in the form of a cubic array of spheres (cub) and as a layered material (tet). The values for the cubic material are obtained using an extended finiteelement simulation procedure $[17,18]$ in which we restrict our attention to $0<f<0.5$ as this range approaches the sphere touching limit and, subsequently, the extent of the numerical procedure. In Figs. 4(a) and 4(b) we observe that values for the layered medium act as approximate bounds for the cubic lattice and suggest that our closed-form expressions may be used to obtain estimates of the photoelastic constants for an arbitrary material pair. The limit behavior of these curves also differs considerably, with only $p_{\mathrm{xxxx}}^{\text {tet }} \approx p_{\mathrm{xxxx}}^{\mathrm{cub}}$ and $p_{\mathrm{xxyy}}^{\mathrm{tet}} \approx p_{\mathrm{xxyy}}^{\mathrm{cub}}$ for vanishing filling fraction. However, we remark that further investigation is needed to determine bounds on the photoelastic properties of composite materials.

\section{CONCLUDING REMARKS}

We have presented an accurate procedure for determining the acousto-optic properties of layered media, fully accounting for artificial photoelasticity and the roto-optic effect. The methods outlined in this work are easily generalizable to layered media with anisotropic constituents. This study opens the path for exploring the acousto-optic properties of highly

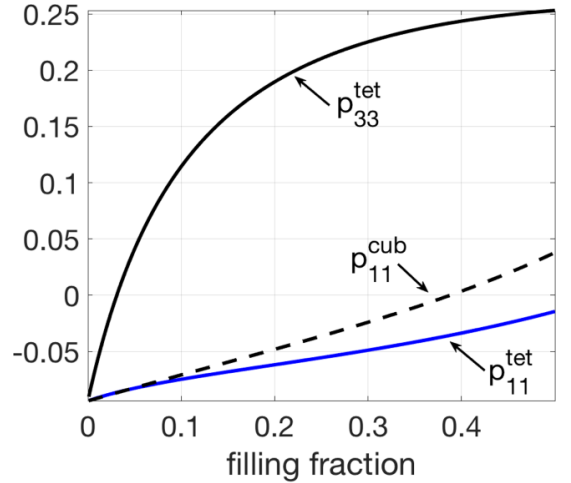

(a)

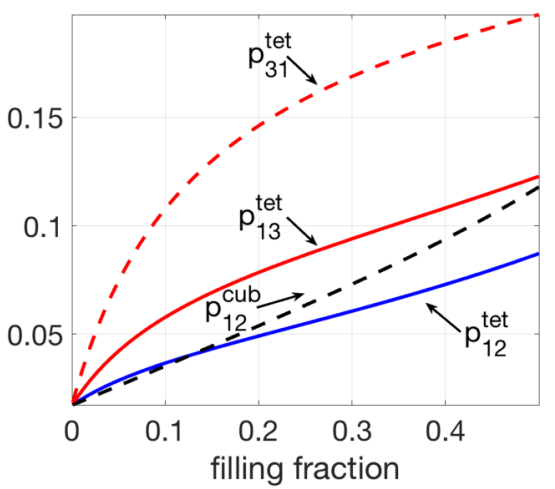

(b)

FIG. 4. Comparison of symmetric photoelastic coefficients (a) $p_{\mathrm{xxxx}}^{\mathrm{eff}}$ and (b) $p_{\mathrm{xxyy}}^{\mathrm{eff}}$ as a function of filling fraction for a cubic array (cub) of $\mathrm{As}_{2} \mathrm{~S}_{3}$ glass spheres embedded in $\mathrm{Si}$ [100] (where $f=$ $4 \pi r^{3} /\left[3\left(a+a^{\prime}\right)^{3}\right]$ and $r$ is the radius of a sphere) with corresponding terms for a layered medium (tet) comprising the same materials [with $\left.f=a /\left(a+a^{\prime}\right)\right]$, with $a+a^{\prime}=50 \mathrm{~nm}$.

anisotropic media, such as hyperbolic metamaterials [36] and thin film composites [15].

We have shown that the symmetric photoelastic constants $p_{i j k l}^{\text {eff }}$ of a layered material are nontrival functions of filling fraction, can exhibit extraordinary enhancement, and can be tuned as desired for applications.

We have also demonstrated that roto-optic coefficients can take values comparable to the symmetric photoelastic coefficients. This result has important implications for acoustic shear-wave propagation in optically anisotropic media. Furthermore, the tunable photoelastic response offered by layered materials may have important implications for SBS structures.

\section{ACKNOWLEDGMENTS}

This work was supported by the Australian Research Council: CUDOS Centre of Excellence Project No. CE110001018 and Discovery Projects No. DP150103611, No. DP160101691.
[1] F. Pockels, Ann. Phys. (Berlin, Ger.) 275, 440 (1890).

[2] D. F. Nelson and M. Lax, Phys. Rev. B 3, 2778 (1971).

[3] D. F. Nelson, Electric, Optic, and Acoustic Interactions in Dielectrics (Wiley, New York, 1979).

[4] R. W. Dixon, J. Appl. Phys. 38, 5149 (1967).
[5] B. J. Eggleton, C. G. Poulton, and R. Pant, Adv. Opt. Photonics 5, 536 (2013).

[6] R. E. Newnham, Properties of Materials: Anisotropy, Symmetry, Structure: Anisotropy, Symmetry, Structure (Oxford University Press, New York, 2004). 
[7] M. Aspelmeyer, T. J. Kippenberg, and F. Marquardt, Rev. Mod. Phys. 86, 1391 (2014).

[8] B. Djafari-Rouhani, S. El-Jallal, and Y. Pennec, C. R. Phys. 17, 555 (2016).

[9] A. Kobyakov, M. Sauer, and D. Chowdhury, Adv. Opt. Photonics 2, 1 (2010).

[10] P. E. Powers, Fundamentals of Nonlinear Optics (CRC Press, Boca Raton, Florida, 2011).

[11] D. K. Biegelsen, Phys. Rev. Lett. 32, 1196 (1974).

[12] R. Van Laer, B. Kuyken, D. Van Thourhout, and R. Baets, Nat. Photonics 9, 199 (2015).

[13] E. Peral and A. Yariv, IEEE J. Quantum Elect. 35, 1185 (1999).

[14] J. E. Sipe and R. W. Boyd, Phys. Rev. A 46, 1614 (1992).

[15] G. W. Milton, The Theory of Composites (Cambridge University Press, Oxford, 2002).

[16] M. J. A. Smith, B. T. Kuhlmey, C. M. de Sterke, C. Wolff, M. Lapine, and C. G. Poulton, Phys. Rev. B 91, 214102 (2015).

[17] M. J. A. Smith, B. T. Kuhlmey, C. M. de Sterke, C. Wolff, M. Lapine, and C. G. Poulton, Opt. Lett. 41, 2338 (2016).

[18] M. J. A. Smith, B. T. Kuhlmey, C. M. de Sterke, C. Wolff, M. Lapine, and C. G. Poulton, J. Opt. Soc. Am. B 33, 2162 (2016).

[19] B. D. Rouhani and J. Sapriel, Phys. Rev. B 34, 7114 (1986).

[20] D. F. Nelson and M. Lax, Phys. Rev. Lett. 24, 379 (1970).

[21] J. F. Nye, Physical Properties of Crystals: Their Representation by Tensors and Matrices (Oxford University Press, Oxford, 1985).

[22] J. Sapriel, Acousto-optics (Wiley, Chichester, United Kingdom, 1979).
[23] D. F. Nelson, P. D. Lazay, and M. Lax, Phys. Rev. B 6, 3109 (1972).

[24] C. Wolff, M. J. Steel, B. J. Eggleton, and C. G. Poulton, Phys. Rev. A 92, 013836 (2015).

[25] B. Djafari-Rouhani and E. M. Khourdifi, in Light Scattering in Semiconductor Structures and Superlattices, edited by D. J. Lockwood and J. F. Young (Plenum, New York, 1991), pp. 139-158.

[26] O. Matsuda and O. B. Wright, J. Opt. Soc. Am. B 19, 3028 (2002).

[27] D. Schneider, F. Liaqat, E. H. El Boudouti, O. El Abouti, W. Tremel, H.-J. Butt, B. Djafari-Rouhani, and G. Fytas, Phys. Rev. Lett. 111, 164301 (2013).

[28] J. Chan, A. H. Safavi-Naeini, J. T. Hill, S. Meenehan, and O. Painter, App. Phys. Lett. 101, 081115 (2012).

[29] Q. Rolland, M. Oudich, S. El-Jallal, S. Dupont, Y. Pennec, J. Gazalet, J. Kastelik, G. Lévêque, and B. Djafari-Rouhani, Appl. Phys. Lett. 101, 061109 (2012).

[30] S. G. Johnson, M. Ibanescu, M. Skorobogatiy, O. Weisberg, J. D. Joannopoulos, and Y. Fink, Phys. Rev. E 65, 066611 (2002).

[31] D. F. Nelson and P. D. Lazay, Phys. Rev. B 16, 4659 (1977).

[32] D. J. Bergman, Phys. Rep. 43, 377 (1978).

[33] M. Grimsditch, Phys. Rev. B 31, 6818 (1985).

[34] J. D. Jackson, Classical Electrodynamics, 2nd ed. (Wiley, New York, 1962).

[35] B. A. Auld, Acoustic Fields and Waves in Solids (Wiley, New York, 1973).

[36] V. Drachev, V. A. Podolskiy, and A. V. Kildishev, Opt. Express 21, 15048 (2013). 